\title{
Vehicle Dynamics Prediction Based on State Observers Entries Anticipation
}

\author{
Felipe H. R. da Cunha, ${ }^{*}$ Alessandro Correa Victorino, ${ }^{*}$ \\ Raymond Ghandour* and Ali Charara* \\ * Heudiasyc Laboratory UMR CNRS 6599, Université de Technologie \\ de Compiègne UTC, BP20529-60205 Compiègne, France \\ (felipe.rodrigues-da-cunha@hds.utc.fr, acorreav@hds.utc.fr, \\ rghandou@hds.utc.fr, acharara@hds.utc.fr)
}

\begin{abstract}
This paper presents a prediction method for vehicle dynamics based on the anticipation of the state observer entries. The methodology consists in adopting hypotheses about the trajectory, velocity and acceleration in a future instant and in using these assumptions, allied to the previous road geometric information, to calculate the future tire/road lateral and vertical efforts using a state observer/anticipation method. Once knowing dynamical behavior in a future maneuver, indexes are calculated, in order to advertise a control system (the driver or an embedded computer) to the possibility of an accident, using: the percentual mobilized adherence and the load transfer ratio parameters.
\end{abstract}

Keywords: Vehicle dynamics, Automotive control, State observers, Drifting evaluation, Rollover evaluation

\section{INTRODUCTION}

Road accidents take place mainly in departmental and national roads making almost 1.3 million deaths and between 20 and 50 millions of injured worldwide every year. Among the reasons that lead to this result, we must highlight the driver's errors (see Aparicio (2005)) - that are commonly traduced in the form of an inappropriate adoption of a speed. This mistaken adoption is generally due to visibility problems and sharp roadway curves that have an inadequate signing policy or even no warning at all. As a consequence, the vehicle is likely to drift and rollover.

On the other hand, looking to dwindle the occurrence of road accidents, advanced driver assistance systems (ADAS) are used. They are designed to help the driver in its driver process and some commons systems are: the adaptive cruise control (ACC), that follows the flow of traffic ahead of the vehicle even if its forward progress is only stop-and-go, the lane departure warning (LDW), that alerts the driver with acoustical warnings before his vehicle is about to leave the lane and the electronic stability control (ESC), that detects loss of steering control and it automatically applies the brakes to help steer the vehicle where the driver intends to go. The latter is responsible of preventing 27 percent of loss-of-control accidents by intervening when emergency situations are detected, as we can see in Hsu and Gerdes (2007), what reflects the importance of these technologies.

Nonetheless the traditional ADAS systems are undoubtedly life-saving technologies, they are not suited to predict rollover and skid situations. In this regard, the proposal of this article is to develop a system that could furnish

\footnotetext{
* This work was supported by CNRS.
}

the driver (or an automatic control system) a warning to these potential situations in a certain electronic horizon. Nowadays, there are systems that propose similar solutions and they are based on different strategies, for example: the method described in Weisshaar (2002) consider only the road geometry to determine the safe speed in the next curve negotiation. While in Glaser et al. (2007), a similar system is proposed taking into account the vehicle dynamics (bicycle model) and the driver behavior (under the form of a coefficient that expresses the value of acceleration the driver is up to accept). Nevertheless, there are also techniques based on knowledge extraction from databases like Mikolajetz et al. (2005), which argues that the parameterizing of such ADAS could be realized with data from realistic, non-critical driving behavior, and Rey and Fogli (2009), where measures are obtained from a "watched" roadway path for modeling human attitudes. However, it seems to be an disadvantage because road monitoring could be expensive and impracticable.

This paper presents a method for predicting the future vehicle dynamics using the estimation obtained with a lateral forces state observer (based on the Dugoff's tire model and Four-Wheel Vehicle model - FWVM) developed by our laboratory. This methodology consists in adopting some reasonable hypotheses for the next curve negotiation and anticipating the observer entries, obtaining the vertical/lateral forces prediction. As a consequence, rollover and drift indexes are determined and the anticipated danger evaluation is made observing their values. These indexes are load transfer parameter (LTR) and percentual mobilized adherence (PMA), they represent the percentual quantity of charge transfered from one car's side to the other and the adherence used by each tire with relation to the maximal value. 
In order to achieve our goal, the work is divided in: the presentation of the general idea, a review of the observer used in this work, see Doumiati et al. (2009), the hypotheses and calculations utilized in the entries anticipation, the risk evaluation methodology, the validation and the results followed by the conclusions. They are respectively organized in sections $2,3,4,5,6$ and 7 .

\section{OVERVIEW OF THE PROPOSED METHODOLOGY}

Figure 1 shows the diagram of the proposed method. This approach assumes that a local map with accurate data is available and the vehicle will roll at the middle of the lane, so the received upcoming curve information is processed to obtain the curvature of the supposed future trajectory. If the safety limits are reached for this optimistic scenario, the algorithm is suitable to predict the most of problems. Besides that, there are several works in trajectory prediction like Broadhurst et al. (2005) and Goyat (2008) but, aiming achieve our primary goals, we will consider this trajectory assumption.

We anticipate the kinematics/dynamics parameters of the car: the wheel steering angle, the yaw rate, the rotational speed of the back tires and the vertical forces $\left(\delta_{i j}, \dot{\psi}, \omega_{2 j}\right.$ and $F z_{i j}$, respectively). They are anticipated considering that the driver will execute the maneuver with the same velocity and acceleration in the longitudinal axis that he is in a time interval before entering the curve. Thus, these physical measures are used as entries for the lateral forces observer that give us the lateral efforts in each wheel.

The knowledge of the lateral and vertical efforts leads to the determination of the security indexes, that expresses the dynamical solicitation with respect to the safety thresholds. The following figure depicts a diagram of the described system (the maximal adherence coefficient calculation block will not be studied in this work).

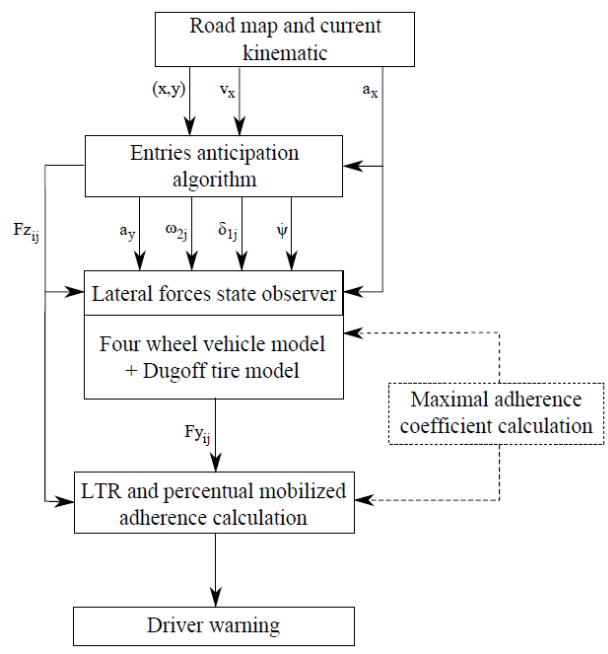

Fig. 1. The algorithm diagram showing the flow of information.

We are proposing a novel method of lateral and normal tire forces prediction based in the anticipation of the observer entries. We also propose the usage of the PMA parameter as a index of drift evaluation. In the next section we will present the lateral forces state observer employed here.

\section{LATERAL FORCES STATE OBSERVER}

The observer uses the Four-Wheel Vehicle model (FWVM), figure 2 .

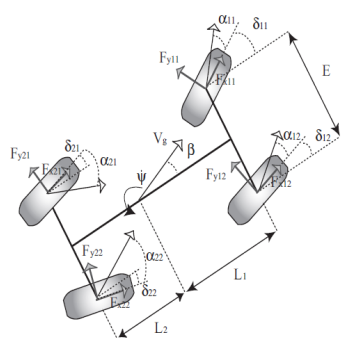

Fig. 2. Four-Wheel Vehicle model.

The lateral dynamics of the vehicle is obtained by summing the forces and moments about the vehicle's center of gravity. Consequently, we have the following dynamic relationships, Doumiati et al. (2009):

$$
\begin{aligned}
\dot{V_{g}}= & \frac{1}{m}\left[F_{x 1} \cos (\beta-\delta)+F_{y 11} \sin (\beta-\delta)+\right. \\
& \left.F_{y 12}(\beta-\delta)+\left(F_{y 21}+F_{y 22}\right) \sin (\beta)\right] \\
\ddot{\psi}= & \frac{1}{I_{z}}\left[l_{f}\left(F_{y 11} \cos \delta+F_{y 12} \cos \delta+F_{x 1} \sin \delta\right]-\right. \\
& l_{r}\left(F_{y 21}+F_{y 22}\right)+\frac{e}{2}\left[F_{y 11} \sin \delta-F_{y 12} \sin \delta\right] \\
\dot{\beta}=- & \dot{\psi}+\frac{1}{m V_{g}}\left[-F_{x 1} \sin (\beta-\delta)+F_{y 11} \cos (\beta-\delta)\right. \\
+ & \left.F_{y 12} \cos (\beta-\delta)+\left(F_{y 21}+F_{y 22}\right) \cos \beta\right] \\
a_{y}= & \frac{1}{m}\left[F_{y 11} \cos \delta+F_{y 12} \cos \delta+\left(F_{y 21}+F_{y 22}\right)+\right. \\
& \left.F_{x 1} \sin \delta\right] \\
a_{x}= & \frac{1}{m}\left[-F_{y 11} \sin \delta-F_{y 12} \sin \delta+F_{x 1} \cos \delta\right]
\end{aligned}
$$

where $m$ is the vehicle mass, $I_{z}$ the yaw moment of inertia, $\delta$ the steering angle and $\delta_{i j}$ the steering angle of each wheel. The steering angle of the rear wheels is zero and we consider the steering angle for the front wheels equals. For that the $\delta$ in the same as $\delta_{1 j}$.

The attempts of modeling the complex interaction between tire and road resulted in many different models based on its physical nature or on empirical conceptualizations. Some examples of these models are Pacejka (2002) and Dugoff et al. (1970). Because of the relative simplicity with respect to the other models, this observer is based on the Dugoff's model. Thus, the nonlinear lateral tire forces are given by:

$$
F_{y i j}=-C_{\alpha i} \tan \left(\alpha_{i}\right) f(\lambda)
$$


where $\alpha_{i j}$ is the tire slip angle, it is the difference between the tire's longitudinal axis and the tire's velocity vector. Its formulation is given by:

$$
\begin{aligned}
& \alpha_{11}=\delta-\arctan \left(\frac{V_{g} \beta+l_{f} \dot{\psi}}{V_{g}-e_{f} \dot{\psi} / 2}\right) \\
& \alpha_{12}=\delta-\arctan \left(\frac{V_{g} \beta+l_{f} \dot{\psi}}{V_{g}+e_{f} \dot{\psi} / 2}\right) \\
& \alpha_{21}=\delta-\arctan \left(\frac{V_{g} \beta-l_{f} \dot{\psi}}{V_{g}-e_{f} \dot{\psi} / 2}\right) \\
& \alpha_{22}=\delta-\arctan \left(\frac{V_{g} \beta-l_{f} \dot{\psi}}{V_{g}+e_{f} \dot{\psi} / 2}\right)
\end{aligned}
$$

and $C_{\alpha i}$ is the lateral stiffness and $f(\lambda)$ is given by:

$$
f(\lambda)=\left\{\begin{array}{l}
(2-\lambda) \lambda ; \quad \lambda<1 \\
1 ; \lambda \geq 1
\end{array}\right.
$$

with:

$$
\lambda=\frac{\mu_{\max } F_{z i j}}{2 C_{\alpha i} \tan \left(\alpha_{i j}\right)}
$$

In equation (5), $\mu_{\max }$ is the maximal adherence coefficient of the road and $F_{z i j}$ is the normal load on the tire. This simplified tire model assumes no longitudinal forces, a uniform pressure distribution, a rigid tire carcass, and a constant coefficient of friction of sliding rubber.

Using the above described model, the observer was implemented in a first-order Euler approximation discrete form employing the unscented Kalman Filter (UKF). Finally, this algorithm was embedded in an experimental vehicle in order to perform its validation in real time Doumiati et al. (2009).

The next section will present the proposed entries anticipation method for this observer showed in figure 1 .

\section{ANTICIPATION OF THE OBSERVER ENTRIES}

In order to anticipate the entries of the observer, it will be considered along this section the following hypotheses:

- The driver will perform a trajectory in the center of the lane.

- The tires will be operating at the linear region.

- The vehicle's longitudinal velocity and acceleration in the maneuver will be equal to the average of these values in one window before the cornering.

There are several works in the trajectory prediction, but in order to achieve our primary goals, we will consider this trajectory assumption. The choice of the third hypothesis is in order to warn the driver that if he will continue with the same dynamics as a window before the cornering, he will be in a dangerous situation while passing the cornering. The next developments describe the entries anticipation in accordance with Figure 1.

\subsection{Vertical forces $\left(F z_{i j}\right)$}

Considering available the position of the upcoming road (for example provided by a digital map), the lane center could be calculated and it is given by the Cartesian coordinates $(x, y)$. Thus, the trajectory curvature is approached by:

$$
k=\frac{\frac{d^{2} y}{d x^{2}}}{\left[1+\left(\frac{d y}{d x}\right)^{2}\right]^{\frac{3}{2}}}
$$

As a result, the lateral acceleration, is anticipated as showed in the following equation.

$$
a_{y}=v_{x}^{2} k
$$

where $v_{x}$ is the longitudinal velocity obtained in accordance with the third hypothesis given above.

Employing the result of equation 7, we can finally found the predicted vertical forces using the torque summatory from the roll dynamics, see Doumiati et al. (2008):

$$
\begin{aligned}
F z_{f l, f r} & =\frac{1}{2} m_{v}\left(\frac{l_{r}}{l} g-\frac{h}{l} a_{x}\right) \pm m_{v}\left(\frac{l_{r}}{l} g-\frac{h}{l} a_{x}\right) \frac{h}{e_{f} g} a_{y} \\
F z_{r l, r r} & =\frac{1}{2} m_{v}\left(\frac{l_{r}}{l} g-\frac{h}{l} a_{x}\right) \pm m_{v}\left(\frac{l_{r}}{l} g-\frac{h}{l} a_{x}\right) \frac{h}{e_{r} g} a_{y}
\end{aligned}
$$

where: $l_{f}$ and $l_{r}$ are the distances from the COG (center of gravity) to the front and rear axles, $l$ the total latitudinal distance, $e_{f}$ and $e_{r}$ the front and rear vehicleâs track, $h$ the height of the center of gravity, $m_{v}$ the total mass of the vehicle, $a_{x}$ the longitudinal acceleration and $g$ the gravitational constant.

\subsection{Yaw rate $(\dot{\psi})$}

The yaw rate is defined as the vehicle's angular velocity about its yaw axis, or rate of change of heading. In a car the yaw axis is the "spin" degree of freedom and it can be mathematically defined as a function of the trajectory as showed in the following equation:

$$
\dot{\psi}=\frac{d}{d t}\left[t g^{-1}\left(\frac{d y}{d x}\right)\right]
$$

\subsection{Steering angle $\left(\delta_{1 j}\right)$}

Ackermann steering geometry is a geometric arrangement of linkages in the steering of a car or other vehicle designed to solve the problem of wheels on the inside and outside of a turn needing to trace out circles of different radius. However, modern cars do not use pure Ackermann steering, partly because it ignores important dynamic and compliant effects, we are going to use this simplified model once that the influence of the same is not so important in the final estimations.

$$
\delta_{\text {in } / \text { out }}=\frac{1}{k^{-1} \pm e_{f} / 2}
$$

The internal and external angles are approximately equal. 


\subsection{Rotational speed of the back wheels $\left(\omega_{2 j}\right)$}

Finally, using the integrals of vehicle longitudinal, lateral, and yaw accelerations, the velocity of each wheel hub in the rolling direction of that wheel, can be derived (Osborn and Shim (2006)):

$$
\omega_{2 j}=\frac{v_{x} \pm \dot{\psi} e_{f} / 2}{2 \pi k^{-1}}
$$

As we previously discussed, computing 8, 9, 10 and 11 and feeding the lateral efforts observer with these values, we anticipate $F_{y}$. The next section depicts how to interpret the predicted dynamics to predict danger situations.

\section{DRIFT AND ROLLOVER FUTURE EVALUATION}

In the last section, we have presented the methodology behind the entries anticipation of the lateral forces state observer. Now, we are going to evaluate the drift and rollover danger using the predicted dynamics $\left(F_{z}\right.$ and $\left.F_{y}\right)$. This evaluation is made through the analysis of the danger indexes that will be described in this section.

\subsection{Load Transfer Ratio - LTR}

The LTR parameter is a measure of how much charge have been transfered from one side to the other of the vehicle and it is defined in Boettiger et al. (2003) as:

$$
L T R=\frac{F_{z l}-F_{z r}}{F_{z l}+F_{z r}}
$$

The value of LTR varies from -1 at the lift-off of the left wheels, tends toward 0 at no load transfer, and to 1 at the lift-off of the right wheels.

\subsection{Percentual Mobilized Adherence - PMA}

On the other hand, we can also evaluate the quantity of mobilized adherence with respect to the available amount. So, consider the adherence coefficient definition when the lateral efforts are much greater than the longitudinal ones.

$$
\mu_{i j}=\frac{F_{y i j}}{F_{z i j}}
$$

Considering that the road maximal friction coefficient $\mu_{\max }$ is available, using for instance the methods described by Ghandour et al. (2010), Tanelli et al. (2008) or Muller et al. (2003), the percentual mobilized adherence (PMA) is defined by:

$$
P M A_{i j}=\frac{\mu_{i j}}{\mu_{\max }}
$$

Actually, the PMA reflects the distance between the predicted tire operation point with relation to the road maximal friction coefficient, that signals the beginning of the saturation operation region. In this region the vehicle is considered to be in a dangerous situation. The following picture depicts this paragraph.

For both 12 and 14 parameters, we will adopt a safety limit of $80 \%$, in other words, if the vehicle reach $80 \%$ of load transfer or mobilized adherence, it will be considered in a dangerous situation. The $80 \%$ threshold is adjusted to give the driver the time to correct the situation before the accident.

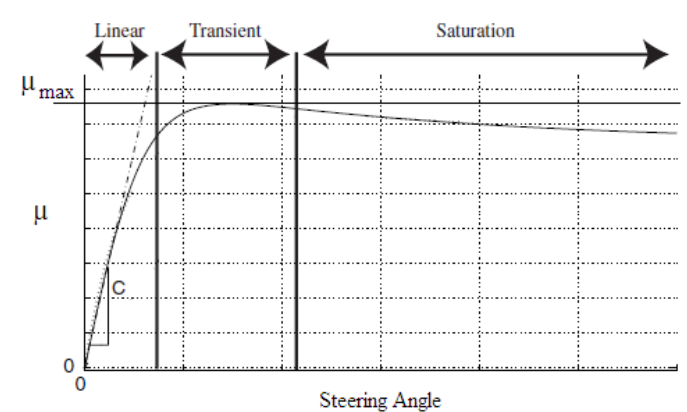

Fig. 3. Tire operation regions

\section{VALIDATION AND RESULTS}

In this section we will present an off-line validation of the proposed method using the data collected from the CALLAS simulator. This software is a realistic simulator validated by vehicle manufacturers and research institutions. The CALLAS model takes into account vertical dynamics (suspension, tires), kinematics, elasto-kinematics, tire adhesion and aerodynamics.

For this validation we have chosen a clothoïdal test track. The clothoïd is a transcendental plane curve which its curvature varies linearly with its curvilinear abscissa. In addition, the main property of this curve is that the centrifugal force experienced by a vehicle traveling continuously along a clothoïdal track varies continuously. For this reason, the clothoid is the most comfortable way to introduce a maneuver and, consequently, widely used in highways shapes. The picture 4 depicts the adopted track.

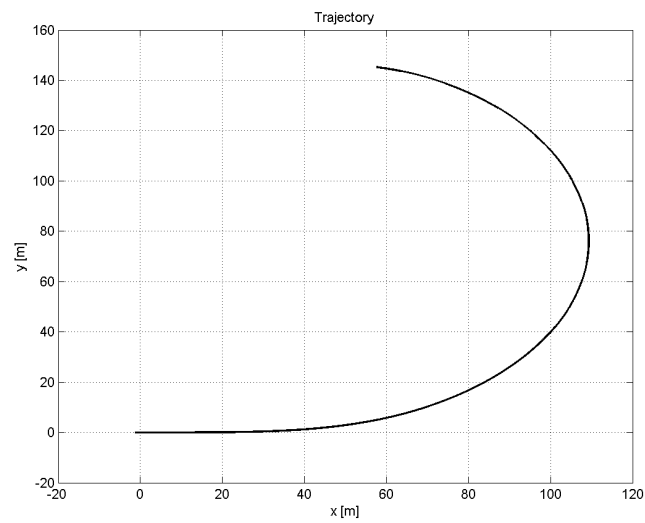

Fig. 4. Trajectory

The simulation was performed with a standard car riding at a constant speed of $80 \mathrm{Km} / \mathrm{h}$. Figure 5 presents the longitudinal-lateral accelerations diagram obtained.

The acceleration diagram, Figure 5, shows that large lateral accelerations were obtained (absolute value up to $0.6 \mathrm{~g}$ ) improving that the experimental vehicle was put in a critical driving situation.

Using the anticipation method described in section IV, we obtained the normal and lateral forces predictions showed in figures 6 and 7 . The results are compared with the values given by CALLAS (considered as real values here). 


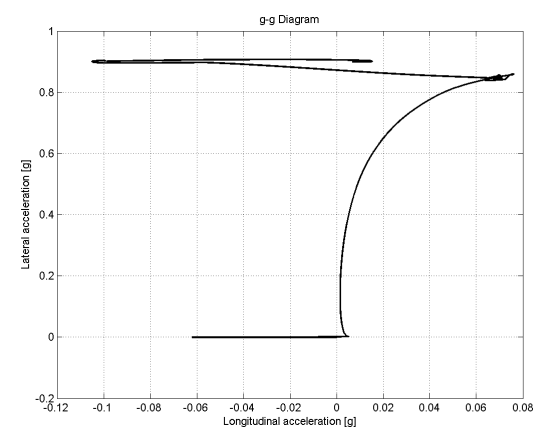

Fig. 5. Longitudinal-Lateral accelerations diagram
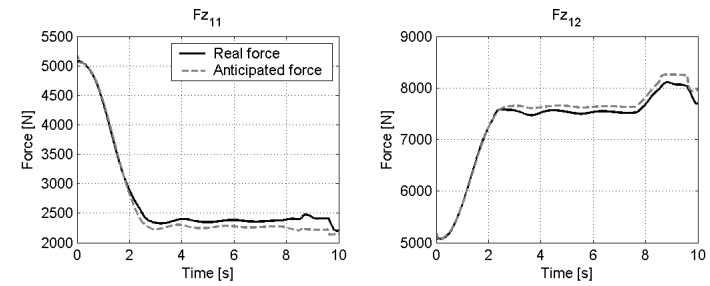

$\mathrm{Fz}_{21}$
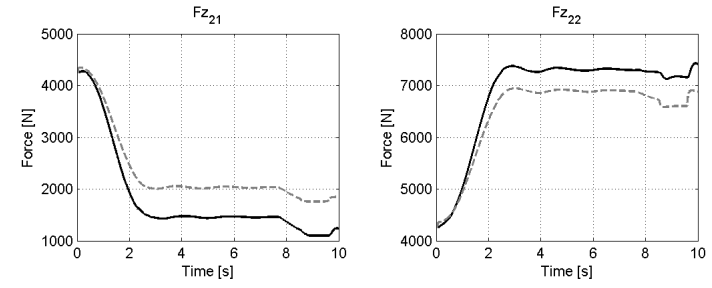

Fig. 6. Comparison between the predicted and simulated normal forces
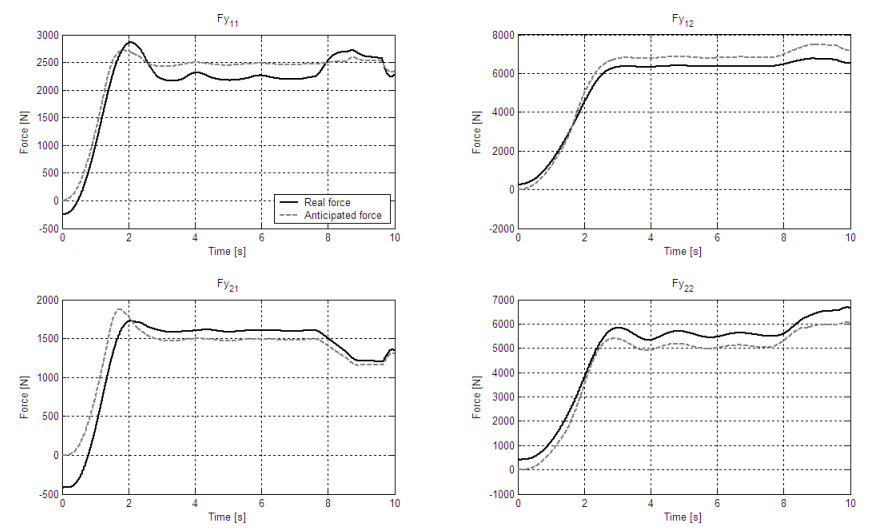

Fig. 7. Comparison between the predicted and simulated lateral forces

Accordingly with section $\mathrm{V}$, we analyze the indexes defined by 14 and 12 (Figures 8 and 9) to evaluate the lateral adherence loss and rollover.

The Figure 8 shows the predicted mobilized adherence in each tire and the maximal road friction $\left(\mu_{\max }=1.1\right.$, dry road). Furthermore, the gray part of the curve corresponds to the predictions in which the car will need more than $80 \%$ of the available tire/road adherence to be kept on the road and for these predictions the system will furnish an alert.

On the other hand, we also have the LTR coefficient (Figure 9).
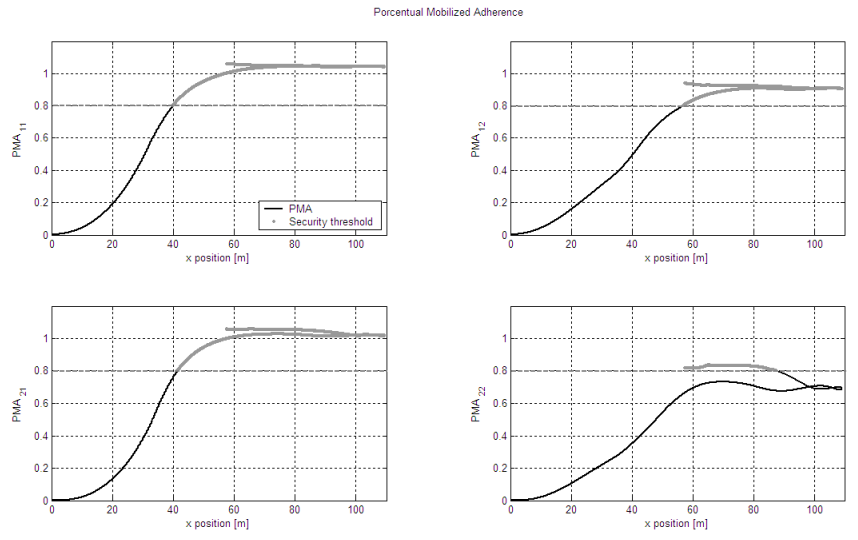

Fig. 8. Absolute adherence in each tire

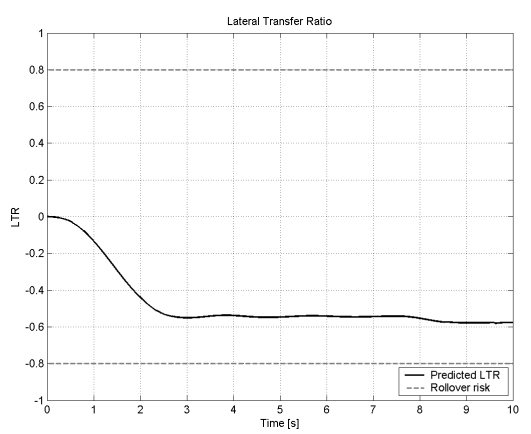

Fig. 9. Load transfer ratio

As a matter of fact, small vehicles tend to lost adherence before rolling over due to their low center of gravity, for this reason the LTR does not assume dangerous values (the lateral load transfer ratio does not exceed 80\%) for this test. However, it is still important to evaluate this index once that we are interested in proposing a generic sytem that could be embedded in different vehicles (e.g. school buses, utility vehicles, trucks) simply changing the observer parameters.

Finally, the alert generation as a function of the future car position is presented in figure 10 .

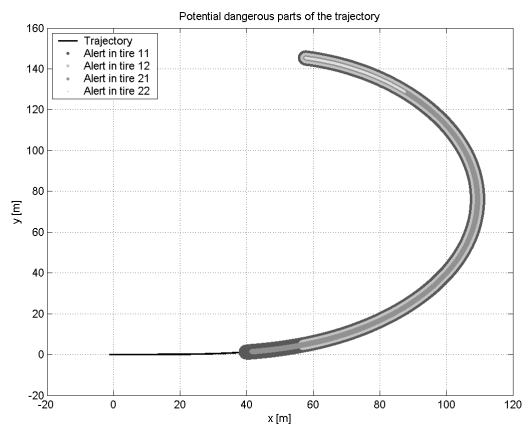

Fig. 10. Alert generation as a function of the future car position

\section{CONCLUSIONS}

This paper presented a dynamics prediction system based on the entries anticipation of the lateral efforts state 
observer. The importance of dynamics prediction lies in the rollover and adherence loss future evaluation, made through the observation of the values assumed by the LTR and PMA (also proposed in this article) parameters. In this way, this algorithm can predict a dangerous situation and provide an information to avoid a possible accident: a driver warning or an entry for an autonomous speed limitation control system.

Moreover, the PMA parameter was also proposed in this article. This index quantifies the percentual distance between the predicted adherence coefficient with relation to the maximal tire/road friction coefficient. This quantity signs which operation region the tires will work in the prediction horizon. If the PMA assumes a value superior to 0.8 , it means that the vehicle is leaving the linear region and consequently operating in a non-safe region (saturation region). We assume that the maximal road coefficient is known using some method cited earlier.

We have also performed an off-line validation of our system using the CALLAS simulator. We used a standard car riding at $80 \mathrm{Km} / \mathrm{h}$ in a clothoïdal test track. As we saw in section VI, the proposed objective was reached and the system was able to predict a dangerous situation. However, because the LTR parameter did not assume critical values (low center of gravity of the test car), the warning was restricted to drift situation.

The prospects to this work are:

- the development of a robust anticipation method;

- the integration of GPS measurements to have the road configuration;

- the integration of a maximum friction estimation method;

- the implementation of another factors like road inclination and driver behavior;

- and finally the validation by embedding the system in the experimental vehicle.

The experimental vehicle shown in the figure below is the HEUDIASYC laboratory's test vehicle. It is a Peugeot 308 equipped with several sensors including accelerometers, gyrometers, steering angle sensors, linear relative suspension sensors, three correvits and two dynamometric wheels (Figure 11). The dynamometric wheels are able to measure tire forces and wheel torques in and around all three dimensions, provides the information used to compare with the predicted forces.

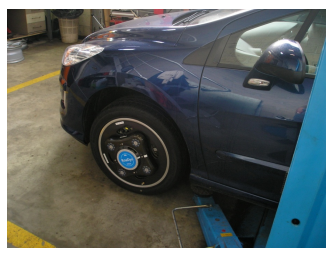

Fig. 11. Experimental vehicle

Finally, the discussed system works taking advantage of the hardware (standard instrumentation measurement available at the CAN bus) and the software (lateral forces state observer) of the test vehicle.

\section{REFERENCES}

Aparicio, F. (2005). Discussion of a new adaptive speed control system incorporating the geometric characteristics of the road. Int. J. Vehicle Autonomous Systems, $3(1), 47-64$.

Boettiger, F., Hunt, K., and Kamnik, R. (2003). Roll dynamics and lateral load transfer estimation in articulated heavy freight vehicles. Proc. Instn Mech. Engrs, Vol. 217 Part D: J.

Broadhurst, A.E., Baker, S., and Kanade, T. (2005). Monte carlo road safety reasoning. presented at the IEEE Intelligent Vehicle Symposium (IV2005), IEEE., $319-324$.

Doumiati, M., Victorino, A., Charara, A., and Lechner, D. (2008). An estimation process for vehicle wheel-ground contact normal forces. IFAC WC08, Seoul Korea.

Doumiati, M., Victorino, A., Charara, A., and Lechner, D. (2009). Unscented kalman filter for real-time vehicle lateral tire forces and sideslip angle estimation. Intelligent Vehicles Symposium IEEE, 45, 901 - 906.

Dugoff, J., Fanches, P., and Segel, L. (1970). An analysis of tire properties and their influence on vehicle dynamic performance. SAE paper (700377).

Ghandour, R., Victorino, A., Charara, A., and Doumiati, M. (2010). Estimation du coefficient dadherence maximale pneumatique chaussee appliquee a la securite routiere. Conference International Francophone $d A u$ tomatique.

Glaser, S., Nouveliere, L., and Lusetti, B. (2007). Speed limitation based on an advanced curve warning system. Proceedings of the IEEE Intelligent Vehicles Symposium Istanbul, Turkey, June 13-15, 1, 4244-1068.

Goyat, Y. (2008). Estimation precise des trajectoires de vehicule par un systeme optique. Ph.D. thesis, Universite Baise Pascal.

Hsu, Y. and Gerdes, J.C. (2007). Experimental studies of using steering torque under various road conditions for sideslip and friction estimation. Proceedings of the IFAC Symposium on Advances in Automotive Control, Monterey, California.

Mikolajetz, A., Henning, M.J., Tenzer, A., Zobel, R., Krems, J.F., and Petzoldt, T. (2005). Curve negotiation: identifying driver behaviour around curves with the driver performance database. Proceedings of the Fifth International Driving Symposium on Human Factors in Driver Assessment, Training and Vehicle Design.

Muller, S., Uchanski, M., and Hedrick, K. (2003). Estimation of the maximum tire-road friction coefficient. Journal of Dynamic Systems, Measurement, and Control, 125, 607-617.

Osborn, R.P. and Shim, T. (2006). Independent control of all-wheel-drive torque distribution. Vehicle system dynamics, 7, 529-546.

Pacejka, H.B. (2002). Tyre and vehicle dynamics. Elsevier.

Rey, G. and Fogli, D.C.M. (2009). Towards a probabilistic alert procedure of roadway departure. NHTSA's traffic safety behaviorial research studies and reports.

Tanelli, M., Piroddi, L., Piuri, M., and Savaresi, S.M. (2008). Real-time identification of tire-road friction conditions. 17th IEEE International Conference on Control Applications.

Weisshaar, T. (2002). Mapping the road to safety. Mercedes Online Magazine. 